\title{
The Role of 18F-Fluorodeoxyglucose Positron Emission Tomography in the Prognostication, Diagnosis, and Management of Thyroid Carcinoma
}

\author{
Brian Hung-Hin Lang \\ Division of Endocrine Surgery, Department of Surgery, The University of Hong Kong, Queen Mary Hospital, \\ Pokfulam Road, Hong Kong \\ Correspondence should be addressed to Brian Hung-Hin Lang, blang@hkucc.hku.hk
}

Received 22 July 2011; Accepted 15 August 2011

Academic Editor: Yasuhiro Ito

Copyright () 2012 Brian Hung-Hin Lang. This is an open access article distributed under the Creative Commons Attribution License, which permits unrestricted use, distribution, and reproduction in any medium, provided the original work is properly cited.

18F-fluorodeoxyglucose positron emission tomography (FDG-PET) plays an increasingly important role in the prognostication, diagnosis, and management of thyroid carcinoma. For patients diagnosed with primary or persistent/recurrent thyroid carcinoma, a finding of FDG-PET positivity implies a more aggressive tumor biology and a distinct mutational profile, both of which carry prognostic significance. Therefore, FDG-PET positivity may be a useful potential risk factor for preoperative risk stratification in primary thyroid carcinoma. This information may help in the planning of subsequent treatment strategy such as the extent of thyroidectomy, prophylactic central neck dissection, and radioiodine ablation. FDG-PET scan has also been found to be a useful adjunct in characterizing indeterminate thyroid nodules on fine needle aspiration cytology. However, larger-sized prospective studies are required to validate this finding. FDG-PET or FDG-PET/CT scan has become the imaging of choice in patients with a negative whole-body radioiodine scan, but with an abnormally raised thyroglobulin level after total thyroidectomy and radioiodine ablation.

\section{Introduction}

18F-fluoro-2-deoxy-D-glucose-positron emission tomography (FDG-PET) scan is increasingly being used not only in oncology but also in some nononcological specialties, such as neurology, cardiology, and infectious diseases [1]. The fundamental principle of FDG-PET scan is that the nuclide, 18FFDG has two parts, namely, the vector part (i.e., 2-deoxy-Dglucose) and the positron emitting nuclide part (i.e., 18F), and when it gets preferentially taken up by rapidly dividing cells (i.e., malignant cells), it gets "trapped" within the cells and emits positron radiation which is then detected by the scintigraphy [2]. This is called metabolic trapping and this forms the basis for FDG-PET scanning. In oncology, FDGPET has many important clinical applications including initial cancer staging as well as monitoring tumor response to anticancer therapy in lung, colon, lymphoma, melanoma, esophageal cancer, and head and neck and breast cancer [3].
In neurology and cardiology, FDG-PET is a useful tool for localization of epileptogenic zones inside the brain and is a "gold standard" tool for the detection of myocardial viability, respectively $[4,5]$.

Since the first observation of FDG uptake in metastatic thyroid cancer over 20 years ago [6], there have been growing interests in evaluating the role of FDG-PET scanning in the management of thyroid neoplasms [7]. Also with an increased number of FDG-PET scans now being performed, an increasing number of incidental thyroid lesions (or the socalled thyroid incidentalomas) have been found and this itself poses a diagnostic challenge to clinicians. Given that FDG-PET imaging could provide potentially relevant information on tumor biology and the scan results may enable to prognostically stratify thyroid carcinoma patients, the author believes that it would be both timely and important to examine the prognostic significance of FDG-PET positivity in thyroid carcinoma and the role of FDG-PET in diagnosis 


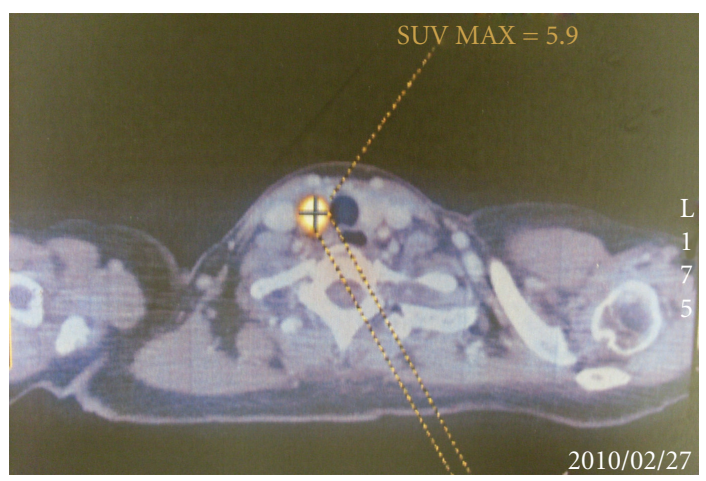

FIGURE 1: A 70-year-old lady underwent 18F-FDG PET for staging of a rectal carcinoma. A focal right thyroid hypermetabolic uptake (with a SUVmax of 5.9) was detected. This was later confirmed as papillary thyroid carcinoma (PTC) on cytology. A total thyroidectomy was performed revealing a $1.3 \mathrm{~cm}$ tall cell variant of PTC with central compartment lymph nodes metastases (pT1N1a).

and management of thyroid carcinoma by a review of the current literature.

\section{Prognostic Significance of FDG-PET Positivity or FDG Avidity in Thyroid Carcinoma}

It has been well recognized that thyroid carcinoma metastases which are shown up on FDG-PET scan do not take up radioactive iodine (RAI) [7]. In fact, it was noted that there was a reciprocal or "flip-flop" relationship between RAI and FDG uptake [6]. Numerous reports have confirmed this important relationship [8]. At the same time, it was noted that those metastases which did not take up RAI were generally less differentiated on histology and behaved clinically more aggressively. Therefore, FDG-PET positivity in thyroid carcinoma could imply clinically more aggressive tumors and poorer overall prognosis. However, before discussing the implications of FDG positivity in thyroid carcinoma, it should be realized that there are fundamental differences in the management of FDG-PET positivity between primary and persistent/recurrent thyroid carcinoma. As a result, the following discussion was subdivided into primary and persistent/recurrent thyroid carcinoma.

\section{Prognostic Significance of FDG-PET Positivity in Primary Thyroid Carcinoma}

Unlike persistent/recurrent carcinoma, the number of studies examining the relationship between FDG-PET positivity and tumor behavior or prognosis for primary thyroid carcinoma remained relatively few. As the role of FDG-PET in preoperative thyroid carcinoma staging remains undefined, the majority of these primary thyroid carcinomas exhibiting FDG-PET positivity are incidentally found cancers or incidentalomas (see Figure 1). Reasons for the lack of enthusiasm for FDG-PET as a staging tool include that FDG-PET scan remains a relatively expensive preoperative imaging modality when compared to ultrasound (USG) which is the recommended imaging modality before surgery, and relative to the other imaging modalities, FDG-PET does not seem to provide any additional staging information (such as the status of the cervical lymph nodes) to an extent of altering the surgical management [9]. As a result, FDG-PET scan has not been routinely advocated as a preoperative staging tool, although there might be a role in selected, more aggressive pathologies, such as Hurthle cell or anaplastic thyroid carcinoma [1012]. Nevertheless, one of the first studies examining the relationship between tumor behavior and FDG-PET positivity in primary thyroid carcinoma was reported by Jeong et al. [13]. In this study, they reported 44 consecutive patients with papillary thyroid microcarcinoma (PTMC) confirmed by USG and fine needle aspiration cytology (FNAC) who subsequently underwent FDG-PET scans before surgery. The clinicopathological characteristics of these 44 PTMC were correlated with the activity of the FDG-PET scan or FDG standardized uptakes values (SUVs). Although there was a strong correlation between SUVs and extrathyroidal extension of PTMC in the univariate analysis, the study did not find any association between the degree of SUV and extrathyroidal extension or other aggressive tumor features in the multivariate analysis [13]. Only age $>45$ and tumor site turned out to be the two significant factors for determining extrathyroidal extension in primary PTMC [13]. SUVs were correlated with tumor size, but this was not unexpected because there is the partial volume effect between smalland large-sized lesions. Therefore, based on this initial study, FDG-PET positivity was not associated with more aggressive tumor behavior or worse tumor characteristics [13]. However, a more recent study found that for PTMCs which were FDG nonavid, they were not only significantly smaller sized tumors but also less frequent perithyroidal tumor invasion and lymphovascular invasion when compared to the PTMCs which were FDG avid [14]. This implies that FDG-PET positivity might be associated with a more aggressive tumor behavior in primary thyroid carcinoma [14]. However, since tumor size is an important factor for FDG-PET positivity as it relates to the partial volume effect, it would be more appropriate to adjust for tumor size in these clinicopathological studies. Yun et al. reported their retrospective study involving 87 patients with a unifocal PTMC who underwent preoperative FDG-PET before total thyroidectomy and central neck dissection [15]. They defined positive FDG uptake in PTMCs as a discernible focal FDG uptake whereas negative FDG as no discernible FDG uptake. All scans were assessed by two experienced nuclear medicine specialists blinded for patients' clinical and pathological variables. In their multivariate analyses, among other factors such as gender, age, and tumor size, FDG-PET positivity was the only significant factor which strongly correlated with extrathyroidal extension $(\mathrm{OR}=5.95$; 95\% CI: 2.13-16.6) and central lymph node metastases in primary PTMC [15]. This result indicates that visual FDGPET positivity in PTMCs is a potential risk factor which could be useful in preoperative risk stratification. One of the potential clinical applications would be to use the preoperative finding of FDG-PET positivity to select patients for more extensive thyroid resection (i.e., hemithyroidectomy versus 
total thyroidectomy in PTMC) or for prophylactic central neck dissection at the time of total thyroidectomy as it is currently only indicated for high-risk tumors [10]. Our previous studies examining the behavior of FDG-avid primary thyroid carcinoma (mostly incidental thyroid carcinoma) also suggested that these tumors are not only larger in size, more likely to be clinically significant $(42.9 \%$ versus $2.9 \%$, $P=0.001)$ but also more aggressive in terms of having higher frequency of tumor bilaterality $(45 \%$ versus $0 \%, P=$ 0.040 ) when compared to non-FDG-avid tumors $[16,17]$. In fact, we advocated a total thyroidectomy even for FDGavid thyroid carcinoma $<1 \mathrm{~cm}$ in diameter because of the high incidence of tumor bilaterality [16]. Therefore, our data would support the fact that FDG-PET positivity implies more aggressive tumor biology and poorer prognosis in primary thyroid carcinoma.

\section{Prognostic Significance of FDG-PET Positivity in Persistent or Recurrent Thyroid Carcinoma}

Numerous studies have found that metastases from thyroid carcinoma which do not concentrate RAI but take up FDG are clinically more aggressive and have poorer tumor differentiation [7]. Esteva et al. studied 50 differentiated thyroid carcinoma (DTC) patients with elevated thyroglobulin ( $\mathrm{Tg}$ ) and negative whole-body scan (WBS) after total thyroidectomy and RAI ablation. All patients underwent a FDG-PET scan one week after the WBS [18]. The authors correlated the postoperative FDG-PET finding and clinicopathological variables of the primary tumor and found that FDG-PET was positive in $32 / 39$ patients with confirmed persistent or recurrent thyroid carcinoma. When compared to the FDGPET-negative group, the FDG-PET-positive group had significantly larger primary tumor size $(2.82 \mathrm{~cm}$ versus $1,72 \mathrm{~cm}$, $P<0.05)$ and these primary tumors were more likely to have capsular invasion $(62.5 \%$ versus $16.7 \%, P<0.05)$ suggesting that more advanced primary tumors were more likely to have FDG-PET-positive recurrences [18]. Rivera et al. studied the histology of the metastases from 70 patients with RAI refractory but FDG-PET-positive recurrences [19]. Of these 70 patients, $33(47.1 \%)$ had poorly DTC, $16(22.9 \%)$ had well DTC, $6(8.6 \%)$ had Hurthle cell carcinoma, and 1 (1.4\%) had anaplastic carcinoma. Based on these findings, the majority of RAI refractory and FDG-PET positive metastases are of a histological aggressive subtype. Interestingly, when the histology of the primary tumor and its subsequent metastases was matched, in most instances, there was a gradual transformation from well-differentiated histology to less-differentiated histology over time, and this might be the reason why even if the primary tumor might not be FDG avid initially, its metastases become FDG avid over time [19]. This phenomenon was somewhat supported by a recent study which found that $B R A F$ mutations represented early events in thyroid carcinogenesis, whereas mutations of PIK3CA and $A K T 1$ were latter events not found in the primary cancers, but in metastases or recurrent cancers [20].
Apart from the direct relationship between FDG-PET positivity and poorer histological differentiation in persistent or recurrent thyroid carcinoma, it was observed that patients with RAI-refractory but FDG-avid metastases were significantly more likely to die from thyroid carcinoma than those with RAI-refractory and non-FDG-avid metastases. This remained true when tumors were matched for TNM stages. Using a Cox proportional hazard model, Robbins et al. found that age $(\mathrm{RR}=1.33 ; 95 \% \mathrm{CI}: 1.08-1.52)$, FDG status (RR $=7.69 ; 95 \% \mathrm{CI}: 2.17-24.4)$, and number of FDG lesions ( $\mathrm{RR}=1.1 ; 95 \% \mathrm{CI}: 1.08-1.15)$ significantly correlated with cancer-specific survivals [21]. Therefore, one could conclude that FDG-PET positivity in persistent/recurrent thyroid carcinoma and the number of FDG lesions are highly prognostic for survival $[21,22]$.

\section{Molecular Basis of FDG-PET Positivity in Thyroid Carcinoma}

Given the fact that mutations in significant oncogenes such as BRAF, TP53 (all of which are able to activate the mitogenactivated protein kinase pathway) are often present in aggressive histological subtypes, it is reasonable to assume that both primary tumors and their metastases which are FDG-PET positive would have a unique mutational profile. RicarteFilho et al. found that $100 \%$ of FDG-PET positive and RAI refractory tumors carried BRAF mutations, whereas in general only $45 \%$ of DTC would carry such mutations [20]. This led to the postulation that perhaps there is a causal relationship between BRAF mutations and FDG-PET positivity. For the lack of RAI uptake in FDG-avid tumors, it was shown for the first time that in a DTC cell line, the conditional activation of $\mathrm{BRAF}^{\mathrm{V} 600 \mathrm{E}}$ tended to downregulate the expression of the sodium iodide symporter (NIS) which is an important ion pump for the transport of iodine across basolateral membrane [23]. This finding was later confirmed in several studies showing that $\mathrm{BRAF}^{\mathrm{V} 600 \mathrm{E}}$ was associated with reduced NIS and NIS mRNA expression [24]. One of the proposed mechanisms through which BRAF represses NIS is by the induction of robust transforming growth factor (TGF) $\beta$ secretion and subsequent activation of TGF $\beta / \mathrm{Smad}$ signaling [25]. However, these findings would only explain why these FDG-avid tumors do not take up RAI. To establish the possible association between BRAF mutations and FDGPET positivity, Durante et al. examined the expression of several key markers of thyrocyte differentiation including NIS, Tg, thyroperoxidase, TSH receptor, transcription factor $P A X 8$, and glucose transporter type I (GLUT1) in 56 papillary thyroid carcinoma (PTC) with $B R A F$ mutations (i.e., $B R A F$-positive), 37 with no $B R A F$ mutations (i.e., $B R A F$-negative), and 8 normal thyroid tissue [26]. Relative to normal thyroid tissue, all markers including GLUT1 in PTCs were reduced, but more importantly, there were additional increases in GLUT1 mRNA when only the BRAF-positive tumors were selected [26]. It is now believed that among the four GLUT isoforms, GLUT1 is the most prevalent isoform responsible for FDG-PET positivity in less-differentiated thyroid carcinomas [27]. 
TABLE 1: A comparison of studies examining the utility of FDG-PET in detecting malignancy in cytologically indeterminate thyroid nodules.

\begin{tabular}{lcclcc}
\hline First author (year) & No. of patients & No. of carcinoma (\%) & Definition of FDG-PET positivity & Sensitivity (\%) & Specificity (\%) \\
\hline Kresnik (2003) [28] & 24 & $9(37.5)$ & Focal uptake with SUV > & 100 & 100 \\
Mitchell (2005) [29] & 24 & $11(45.8)$ & Focal uptake & 60 & 91 \\
de Geus-Oei (2006) [30] & 44 & $6(13.6)$ & Focal uptake & 100 & 66 \\
Sebastianes (2007) [31] & 42 & $11(26.2)$ & Focal uptake & Focal uptake with SUV > 2 & 57 \\
Hales (2008) [32] & 15 & $7(46.7)$ & Area under SUV curve > 175.5 & 100 & 39 \\
Smith (2008) [33] & 23 & $5(21.7)$ & Focal uptake & 100 & 44 \\
Traugott (2010) [34] & $51^{*}$ & $8(15.6)$ & Varied & 94.8 & 59 \\
Vriens (2011) [35] & $225^{\#}$ & $58(25.8)$ & 47.9 \\
\hline
\end{tabular}

FDG-PET: fluorodeoxyglucose positron emission tomography; SUV: standardized uptake value.

*An ongoing prospective trial; \# a meta-analysis.

\section{Role of FDG-PET in the Diagnosis of Thyroid Carcinoma}

Since histologically benign and malignant thyroid lesions do exhibit some differences in SUV, FDG-PET may have an important role in the characterization of thyroid nodules and more specifically in the diagnosis of thyroid carcinoma. However, currently the most cost-effective and accurate way of making the diagnosis of thyroid carcinoma relies on clinical examination, the use of USG, and USG-guided FNAC. Therefore, FDG-PET has not been widely accepted as a diagnostic tool in thyroid carcinoma, but this may change with time as technology improves [36]. One aspect of diagnosis in which FDG-PET scan has shown some promises is in patients with an indeterminate thyroid lesion on FNAC. By definition, an indeterminate FNAC usually includes follicular lesions, Hurthle cell (oncocytic) lesions, atypical cytology, abnormal cytology, or suspicious cytology. This group remains a diagnostic dilemma to clinicians because approximately $20-30 \%$ of these will be malignant, whereas the rest will be benign by pathological examination [37, 38]. However, nearly all patients with indeterminate FNAC would be required to undergo thyroid lobectomy to establish the diagnosis. In other words, surgical resection will prove unnecessary in over $60-70 \%$ of cases. At present, there is no alternative algorithm for a more conservative management for patients with indeterminate FNAC. Convectional USG, computed tomography (CT), and magnetic resonance imaging (MRI) have been previously shown to be of some value, but FDG-PET is yet to be assessed in a large prospective study [34]. Table 1 shows a comparison of series examining the utility of FDG-PET in detecting malignancy in thyroid nodule with indeterminate FNAC. Study size ranged from 15 to 51 patients, but the inclusion criteria varied between studies. One of the larger series was reported by de Geus-Oei et al. [30]. There were 44 patients with inconclusive FNAC (i.e., follicular neoplasm, Hurthle cell neoplasm, atypical cells, or inadequate) and of these, 6 were malignant and the other 38 were benign. In their experience, FDG-PET did not lead any false negatives implying a negative predictive value (NPV) of $100 \%$. All carcinomas demonstrated FDG uptake, but 13 of 38 benign nodules also had FDG uptake. They showed that using routine FDG-PET in their patient cohort could potentially reduce the number of unnecessary lobectomies by $66 \%$ (95\% CI: 49-80\%) if surgery was not advised for those with no uptake in the thyroid gland on FDG-PET imaging [30]. Importantly, no malignancies were missed (i.e., no false negatives), but still there were 13/38 (34.2\%) who underwent unnecessary lobectomies (i.e., false positives). It was interesting to note in their series that the mean SUV was similar between benign and malignant lesions and one of the PTCs actually had SUV as low as 0.9. To improve the false-positive rate, some studies proposed using focal uptake SUV $>2.0$ as the criterion for FDG-PET positivity $[28,32]$. However, for whatever cut-off in SUV, there is always going to be a trade-off between false positives and false negatives. Smith et al. evaluated the association between SUV uptake over time and malignancy in follicular neoplasm by performing serial FDG-PET scans over a 2hour period and measuring the area under the SUV curve (AUC) [33]. They demonstrated no significant difference in AUC, but found a difference in the dynamics of SUV change over time between benign and malignant lesions [32]. Due to the significant overlap in AUC, they concluded that FDG-PET was not able to predict malignancy in a follicular neoplasm [32]. However, since most of these reported series were retrospective in design and the patient selection was not formally standardized, there is a need for a large, welldesigned prospective study. Recently, Traugott et al. reported the results of 51 patients with indeterminate FNAC as an interim analysis of their prospective trial which began in 2004 [34]. Their data suggested that FDG-PET was an accurate diagnostic modality for identifying malignancy in thyroid nodules at least $1 \mathrm{~cm}$ in diameter and with indeterminate FNAC, with 100\% sensitivity and NPV. They concluded that FDG-PET was of value in excluding malignancy in thyroid nodules with indeterminate FNAC. To overcome the spatial resolution limitation of the FDG-PET, their study only included solitary or dominant nodule that measured $\geq 1 \mathrm{~cm}$ on USG. To achieve adequate power, they aimed to recruit 125 patients and results would be available within the next few years. A recent systematic review and metaanalysis evaluated the role of FDG-PET in patients with indeterminate thyroid FNAC [35]. In this meta-analysis, they analyzed over 200 patients and found that the pooled sensitivity, specificity, PPV, and NPV were 95\%, 39\%, 96\%, 


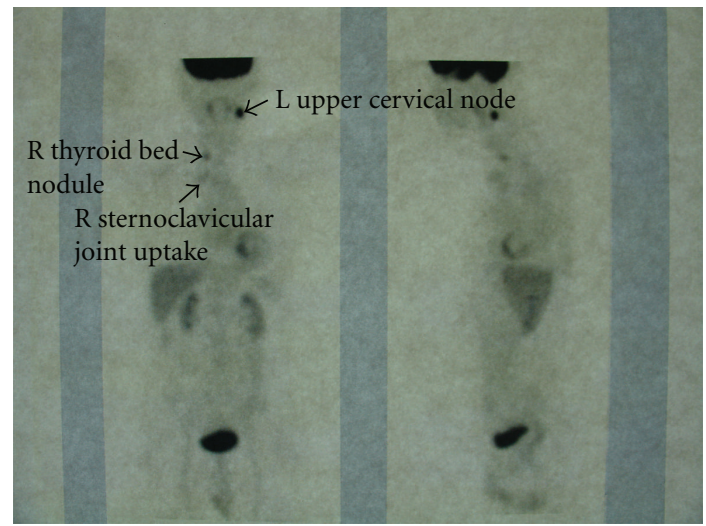

(a)

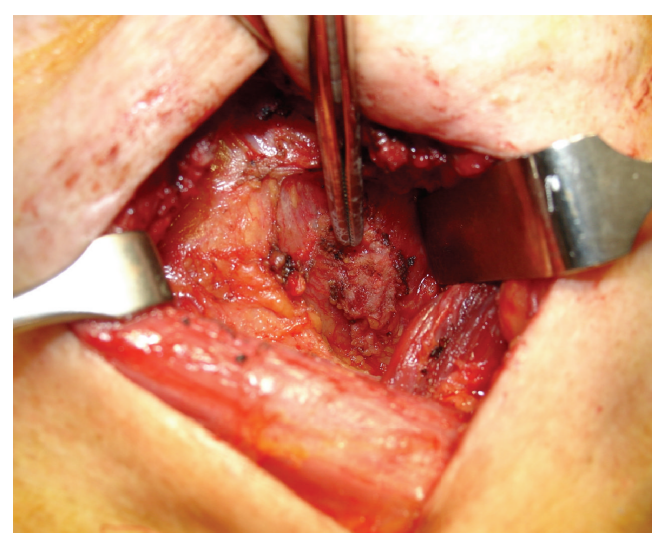

(b)

FiguRE 2: 18F-FDG-PET of a 73-year-old man with an 8-year history of papillary thyroid carcinoma. The serum thyroglobulin was elevated at $66 \mathrm{ug} / \mathrm{L}$ (normal $<55 \mathrm{ug} / \mathrm{L}$ ), but both diagnostic whole-body ${ }^{131}$ I scan and ultrasound were negative for recurrence. (a) FDG-PET revealed hypermetabolic uptakes in left cervical lymph node, right thyroid bed, and right sternoclavicular joint area. (b) An operative picture confirming the presence of tumor recurrence at the right thyroid bed (as pointed by the tip of the forceps).

and $60 \%$, respectively. The authors concluded that a negative FDG-PET scan in patients who had thyroid nodules $>15 \mathrm{~mm}$ with indeterminate FNAB results excluded thyroid cancer [35]. Conversely, a positive FDG-PET result did not identify cancer because approximately $50 \%$ of these patients had benign nodules [35]. They concluded that the incorporation of FDG-PET into the initial workup of such patients before surgery deserves further investigation [35]. However, there are some concerns using FDG-PET in this group of patients. Firstly, follicular thyroid carcinoma (FTC), which accounts for $10-20 \%$ of malignancy in the indeterminate group, is known to be associated with a lower SUV than other histological types of thyroid carcinoma [39]. It is also known that Hurthle cell adenoma, a benign lesion, tends to have very high SUVs [28]. Both entities could possibly lessen the usefulness of FDG-PET in discriminating benign from malignant lesions in this indeterminate group. Furthermore, a confirmatory diagnosis in this group of FNAC is often difficult to make by experienced pathologists. It is known that considerable interobserver and intraobserver variability in the histopathological diagnosis of thyroid follicular lesions has been reported $[40,41]$. In one recent study, among 15 cases of suspected follicular variant of PTC (FVPTC), only 2 cases $(13.3 \%)$ had unanimous agreement among 6 expert pathologists [41]. Given these concerns, the role of FDGPET in thyroid nodules with indeterminate FNAC remains uncertain, but the interim results in one of the possibly largest prospective trials on FDG-PET in cytologically indeterminate nodules looked promising [34]. Hopefully, this prospective trial which is due to complete in the next few years will provide us with more information.

\section{Role of FDG-PET in the Management of Thyroid Carcinoma}

Routine RAI diagnostic scanning or WBS for thyroid carcinoma surveillance is becoming less frequently used because of its relatively low sensitivity and has been supplanted by serum Tg level and neck USG. Nowadays, one of the commonest clinical scenarios would be for a patient with negative or normal USG but raised unstimulated Tg (i.e., $>10 \mathrm{ng} / \mathrm{mL}$ or $10 \mathrm{ug} / \mathrm{L}$ ) [10]. FDG-PET is recommended in patients with suspected recurrence or metastases in the setting of raised $\mathrm{Tg}$ levels and scan-negative metastases (see Figures 2(a) and 2(b)) [10]. Earlier studies found that FDG-PET was a useful diagnostic tool in the followup of postsurgical patients with DTC, negative WBS, and abnormal Tg levels [42]. FDGPET was able to detect metastatic disease over $90 \%$ of cases and more importantly, it was able to change the surgical tactic in a $20-30 \%$ of cases [42]. Similarly, Wang et al. reported their experience of 37 DTC patients with negative WBS and elevated Tg, found that FDG-PET was able to locate occult disease in $71 \%$, and reported a positive predictive value (PPV) of $92 \%$. More importantly, FDG-PET was able to change the clinical management in over $50 \%$ of patients [43]. In the presence of low Tg levels, FDG-PET had the NPV of $93 \%$ [43]. Esteva et al. reported the FDG-PET findings in 50 patients with elevated $\mathrm{Tg}$ and negative WBS and found that FDG-PET was positive in $32 / 39$ (82.1\%) patients with confirmed recurrence and negative in $7 / 11(63.6 \%)$ with no confirmed recurrence. The sensitivity and specificity were $82 \%$ and $64 \%$, respectively [18]. Tumor size and capsular tumor invasion were factors significantly associated with a positive FDG-PET study [18]. They also concluded that FDG-PET was an extremely useful imaging tool in patients with negative WBS and raised Tg [18]. However, the added value of FDG-PET/CT over good-quality conventional imaging modalities such as USG, CT, MRI, and diagnostic WBS in locating recurrent or persistent DTC has recently been questioned mainly of the extra cost with FDG-PET/CT and the associated radiation. Lal et al. recently evaluated the added value of FDG-PET/CT over conventional imaging studies in 20 DTC patients with elevated Tg and negative diagnostic WBS [44]. They found FDG-PET/CT provided 


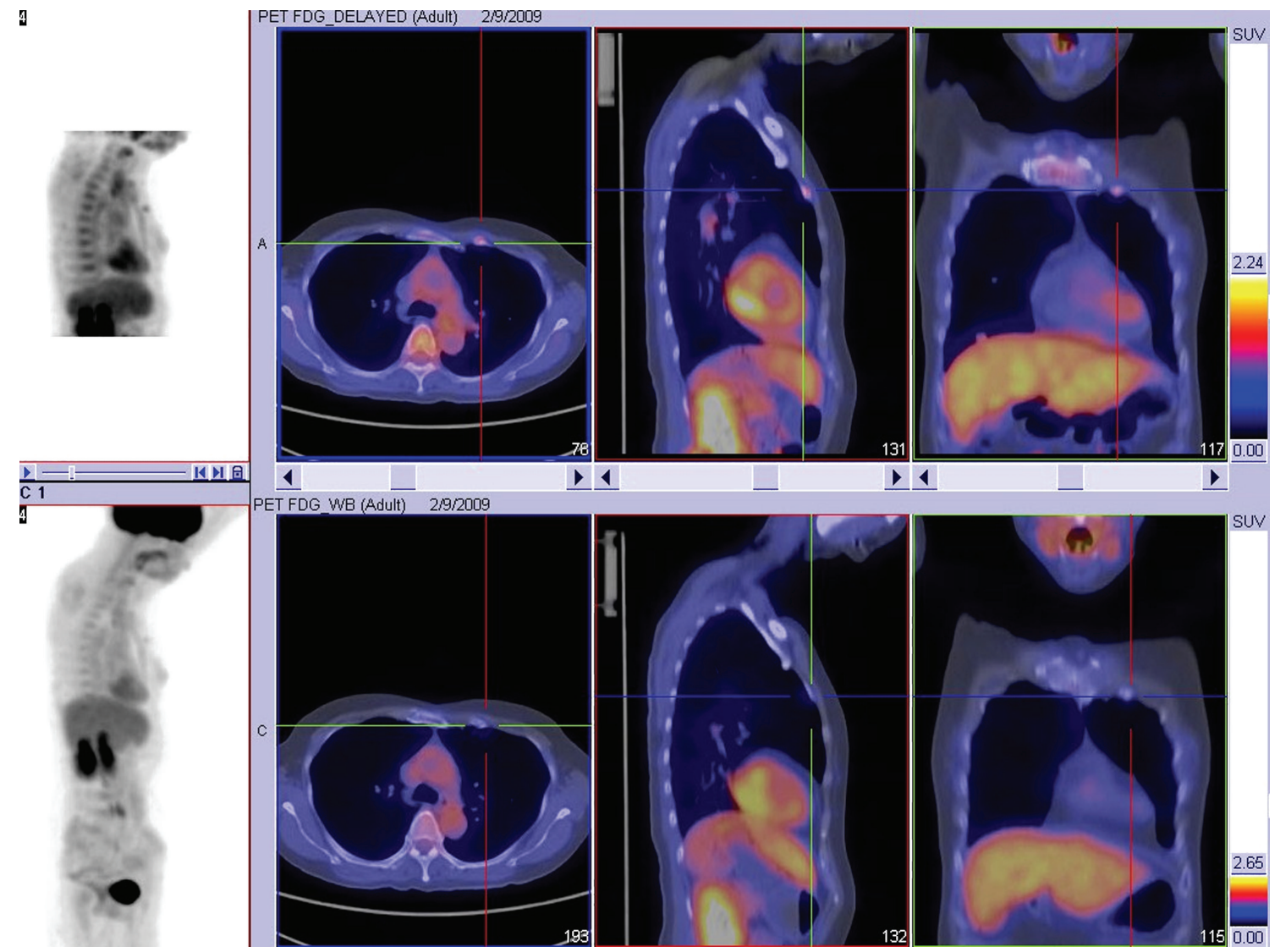

FIGURE 3: 18F-FDG-PET/CT of a 57-year-old woman with a 10-year history of widely invasive follicular thyroid carcinoma. Serum thyroglobulin was elevated to $121 \mathrm{ug} / \mathrm{L}$ (normal < $55 \mathrm{ug} / \mathrm{L}$ ), but both diagnostic whole-body ${ }^{131}$ I scan and ultrasound were negative. The FDGPET/CT revealed hypermetabolic FDG uptakes in the rib cage.

additional information in only $2 / 20(10.0 \%)$ patients, both of whom required no additional intervention, but underestimated the extent of disease in 3/30 (15.0\%) patients and led to unnecessary interventions (including surgery, RAI, and antibiotics) in $3 / 30(15.0 \%)$ additional patients. They concluded that FDG-PET/CT has a good sensitivity in detecting recurrent or persistent DTC, but the added value over good-quality conventional imaging is very limited [44]. Furthermore, it may lead to unnecessary interventions [44]. Perhaps, future large prospective studies are required to resolve some of the controversies.

Some studies evaluated the relationship between TSH level and FDG uptake intensity [45-47]. To date, the evidence seems to suggest that a higher SUVmax could be obtained in TSH-stimulated condition by recombinant human TSH (or rhTSH) stimulation, and as a result of high level of TSH, greater number of FDG-avid metastases could be detected on scanning [45-47]. Some authors evaluated the diagnostic accuracy of integrated FDG-PET/CT as it was believed that fusion of the two modalities may further enhance the sensitivity, specificity, and tumor localization (see Figure 3). For other types of head and neck tumors, combined FDG$\mathrm{PET} / \mathrm{CT}$ has been shown to have improved diagnostic accuracy than FDG-PET or CT alone [48]. Razfar et al. evaluated the diagnostic accuracy of integrity of FDG-PET/CT in detecting recurrent/persistent DTC. They reported the sensitivity, specificity, PPV, and NPV to be $80.7 \%, 88.9 \%, 94.7 \%$, and $65.3 \%$, respectively. From their analysis, they demonstrated there was an alteration in the treatment strategy in $28.2 \%$ as a result of adding the FDG-PET/CT information, and $21 \%$ required additional surgery [49]. Therefore, it would seem that FDG-PET/CT scan might be superior to FDG-PET as the imaging of choice in patients with a negative whole-body radioiodine scan and an abnormally raised thyroglobulin level after total thyroidectomy and radioiodine ablation.

\section{Conclusion}

In patients with either primary or persistent/recurrent thyroid carcinoma, the finding of FDG-PET positivity or FDGavidity usually implies poorer tumor differentiation, more aggressive tumor biology, and worse prognostic outcomes. These observations are supported by the unique mutational profile of FDG-avid tumors or metastases, namely, increased frequency of BRAF mutations leading to decreased NIS and increased GLUT1. FDG-PET positivity may be a useful potential risk factor for preoperative risk stratification in primary thyroid carcinoma and this information may help in the planning of subsequent treatment strategy such as 
the extent of thyroidectomy, prophylactic central neck dissection, and RAI ablation. FDG-PET scan has the potential in characterizing indeterminate thyroid nodules on FNAC. However, larger-sized prospective studies are required to validate this finding. FDG-PET or FDG-PET/CT scan has become the imaging of choice in patients with a negative WBS, but with an abnormally raised Tg level after total thyroidectomy and RAI ablation.

\section{References}

[1] L. Pace, E. Nicolai, M. Klain, and M. Salvatore, "Diagnostic value of FDG PET/CT imaging," The Quarterly Journal of Nuclear Medicine and Molecular Imaging, vol. 53, no. 5, pp. 503-512, 2009.

[2] W. Wadsak and M. Mitterhauser, "Basics and principles of radiopharmaceuticals for PET/CT," European Journal of Radiology, vol. 73, no. 3, pp. 461-469, 2010.

[3] J. W. Fletcher, B. Djulbegovic, H. P. Soares et al., "Recommendations on the use of 18F-FDG PET in oncology," Journal of Nuclear Medicine, vol. 49, no. 3, pp. 480-508, 2008.

[4] A. Newberg, A. Alavi, and M. Reivich, "Determination of regional cerebral function with FDG-PET imaging in neuropsychiatric disorders," Seminars in Nuclear Medicine, vol. 32, no. 1, pp. 13-34, 2002.

[5] B. L. Gerber, F. F. Ordoubadi, W. Wijns et al., "Positron emission tomography using 18F-fluoro-deoxyglucose and euglycaemic hyperinsulinaemic glucose clamp: optimal criteria for the prediction of recovery of post-ischaemic left ventricular dysfunction," European Heart Journal, vol. 22, no. 18, pp. 1691-1701, 2001.

[6] H. Joensuu and A. Ahonen, "Imaging of metastases of thyroid carcinoma with fluorine-18 fluorodeoxyglucose," Journal of Nuclear Medicine, vol. 28, no. 5, pp. 910-914, 1987.

[7] B. H. Lang and T. T. Law, "The role of 18 F-fluorodeoxyglucose positron emission tomography in thyroid neoplasms," The Oncologist, vol. 16, no. 4, pp. 458-466, 2011.

[8] U. Feine, R. Lietzenmayer, J. P. Hanke, H. Wöhrle, and W. Müller-Schauenburg, "18FDG whole-body PET in differentiated thyroid carcinoma. Flipflop in uptake patterns of 18FDG and 131I," Nuklearmedizin, vol. 34, no. 4, pp. 127-134, 1995.

[9] H. S. Jeong, C. H. Baek, Y. I. Son et al., "Integrated 18F-FDG PET/CT for the initial evaluation of cervical node level of patients with papillary thyroid carcinoma: comparison with ultrasound and contrast-enhanced CT," Clinical Endocrinology, vol. 65, no. 3, pp. 402-407, 2006.

[10] D. S. Cooper, G. M. Doherty, B. R. Haugen et al., "Revised American thyroid association management guidelines for patients with thyroid nodules and differentiated thyroid cancer," Thyroid, vol. 19, no. 11, pp. 1167-1214, 2009.

[11] D. A. Pryma, H. Schöder, M. Gönen, R. J. Robbins, S. M. Larson, and H. W. D. Yeung, "Diagnostic accuracy and prognostic value of 18F-FDG PET in Hürthle cell thyroid cancer patients," Journal of Nuclear Medicine, vol. 47, no. 8, pp. 1260-1266, 2006.

[12] T. V. Bogsrud, D. Karantanis, M. A. Nathan et al., "18F-FDG PET in the management of patients with anaplastic thyroid carcinoma," Thyroid, vol. 18, no. 7, pp. 713-719, 2008.

[13] H. S. Jeong, M. Chung, C. H. Baek, Y. H. Ko, J. Y. Choi, and Y. I. Son, "Can [18F]-fluorodeoxyglucose standardized uptake values of PET imaging predict pathologic extrathyroid invasion of thyroid papillary microcarcinomas?" Laryngoscope, vol. 116, no. 12, pp. 2133-2137, 2006.
[14] J. W. Choi, Y. H. Yoon, Y. H. Yoon, S. M. Kim, and B. S. Koo, "Characteristics of primary thyroid carcinoma with falsenegative findings on initial 18F-FDG PET/CT," Annals of Surgical Oncology, vol. 18, no. 5, pp. 1306-1311, 2011.

[15] M. Yun, T. W. Noh, A. Cho et al., "Visually discernible $[18 \mathrm{~F}]$ fluorodeoxyglucose uptake in papillary thyroid microcarcinoma: a potential new risk factor," Journal of Clinical Endocrinology and Metabolism, vol. 95, no. 7, pp. 3182-3188, 2010.

[16] T. T. Law and B. H. H. Lang, "Incidental thyroid carcinoma by FDG-PET/CT: a study of clinicopathological characteristics," Annals of Surgical Oncology, vol. 18, no. 2, pp. 472-478, 2011.

[17] D. K. Chan, B. H. Lang, and T. T. Law, "Value of fluorodeoxyglucose positron emission tomography in characterizing clinically-significant thyroid carcinomas," Surgical Practice, vol. 15, no. 2, pp. 34-40, 2011.

[18] D. Esteva, M. A. Muros, J. M. Llamas-Elvira et al., "Clinical and pathological factors related to $18 \mathrm{~F}-\mathrm{FDG}-\mathrm{PET}$ positivity in the diagnosis of recurrence and/or metastasis in patients with differentiated thyroid cancer," Annals of Surgical Oncology, vol. 16, no. 7, pp. 2006-2013, 2009.

[19] M. Rivera, R. A. Ghossein, H. Schoder, D. Gomez, S. M. Larson, and R. M. Tuttle, "Histopathologic characterization of radioactive iodine-refractory fluorodeoxyglucose-positron emission tomography-positive thyroid carcinoma," Cancer, vol. 113, no. 1, pp. 48-56, 2008.

[20] J. C. Ricarte-Filho, M. Ryder, D. A. Chitale et al., "Mutational profile of advanced primary and metastatic radioactive iodinerefractory thyroid cancers reveals distinct pathogenetic roles for BRAF, PIK3CA, and AKT1," Cancer Research, vol. 69, no. 11, pp. 4885-4893, 2009.

[21] R. J. Robbins, Q. Wan, R. K. Grewal et al., "Real-time prognosis for metastatic thyroid carcinoma based on 2-( 18F)-fluoro2-deoxy-D-glucose-positron emission tomography scanning," Journal of Clinical Endocrinology and Metabolism, vol. 91, no. 2, pp. 498-505, 2006.

[22] D. Deandeis, A. Al Ghuzian, S. Leboulleux et al., "Do histological, immunohistochemical and metabolic (radioiodine and fluorodeoxyglucose uptakes) pattern of metastatic thyroid cancer correlate with patient outcome," Endocrine-Related Cancer, vol. 18, pp. 159-169, 2011.

[23] N. Mitsutake, J. A. Knauf, S. Mitsutake, C. Mesa Jr., L. Zhang, and J. A. Fagin, "Conditional BRAFV600E expression induces DNA synthesis, apoptosis, dedifferentiation, and chromosomal instability in thyroid PCCL3 cells," Cancer Research, vol. 65, no. 6, pp. 2465-2473, 2005.

[24] C. Romei, R. Ciampi, P. Faviana et al., "BRAFV600E mutation, but not RET/PTC rearrangements, is correlated with a lower expression of both thyroperoxidase and sodium iodide symporter genes in papillary thyroid cancer," Endocrine-Related Cancer, vol. 15, no. 2, pp. 511-520, 2008.

[25] G. Riesco-Eizaguirre, I. Rodríguez, A. De la Vieja et al., "The BRAFV600E oncogene induces transforming growth factor beta secretion leading to sodium iodide symporter repression and increased malignancy in thyroid cancer," Cancer Research, vol. 69, no. 21, pp. 8317-8325, 2009.

[26] C. Durante, E. Puxeddu, E. Ferretti et al., "BRAF mutations in papillary thyroid carcinomas inhibit genes involved in iodine metabolism," Journal of Clinical Endocrinology and Metabolism, vol. 92, no. 7, pp. 2840-2843, 2007.

[27] R. Ciampi, A. Vivaldi, C. Romei et al., "Expression analysis of facilitative glucose transporters (GLUTs) in human thyroid carcinoma cell lines and primary tumors," Molecular and Cellular Endocrinology, vol. 291, no. 1-2, pp. 57-62, 2008. 
[28] E. Kresnik, H. J. Gallowitsch, P. Mikosch et al., "Fluorine18-fluorodeoxyglucose positron emission tomography in the preoperative assessment of thyroid nodules in an endemic goiter area," Surgery, vol. 133, no. 3, pp. 294-299, 2003.

[29] J. C. Mitchell, F. Grant, A. R. Evenson et al., "Preoperative evaluation of thyroid nodules with ${ }^{18}$ FDG-PET/CT," Surgery, vol. 138, no. 6, pp. 1166-1175, 2005.

[30] L. F. De Geus-Oei, G. F. F. M. Pieters, J. J. Bonenkamp et al., ${ }^{~}{ }^{18}$ F-FDG PET reduces unnecessary hemithyroidectomies for thyroid nodules with inconclusive cytologic results," Journal of Nuclear Medicine, vol. 47, no. 5, pp. 770-775, 2006.

[31] F. M. Sebastianes, J. J. Cerci, P. H. Zanoni et al., "Role of ${ }^{18} \mathrm{~F}$-fluorodeoxyglucose positron emission tomography in preoperative assessment of cytologically indeterminate thyroid nodules," Journal of Clinical Endocrinology and Metabolism, vol. 92, no. 11, pp. 4485-4488, 2007.

[32] N. W. Hales, G. A. Krempl, and J. E. Medina, "Is there a role for fluorodeoxyglucose positron emission tomography/computed tomography in cytologically indeterminate thyroid nodules?" American Journal of Otolaryngology, vol. 29, no. 2, pp. 113$118,2008$.

[33] R. B. Smith, R. A. Robinson, H. T. Hoffman, and M. M. Graham, "Preoperative FDG-PET imaging to assess the malignant potential of follicular neoplasms of the thyroid," Otolaryngology-Head and Neck Surgery, vol. 138, no. 1, pp. 101-106, 2008.

[34] A. L. Traugott, F. Dehdashti, K. Trinkaus et al., "Exclusion of malignancy in thyroid nodules with indeterminate fine-needle aspiration cytology after negative $18 \mathrm{f}$-fluorodeoxyglucose positron emission tomography: interim analysis," World Journal of Surgery, vol. 34, no. 6, pp. 1247-1253, 2010.

[35] D. Vriens, J. H. de Wilt, G. J. van der Wilt, R. T. Netea-Maier, W. J. Oyen, and L. F. de Geus-Oei, "The role of [(18) F]2-fluoro-2-deoxy-d-glucose-positron emission tomography in thyroid nodules with indeterminate fine-needle aspiration biopsy: systematic review and meta-analysis of the literature," Cancer. In press.

[36] B. H. H. Lang and C. Y. Lo, "Technological innovations in surgical approach for thyroid cancer," Journal of Oncology, vol. 2010, Article ID 490719, 6 pages, 2010.

[37] A. D. Bloom, L. P. Adler, and J. M. Shuck, "Determination of malignancy of thyroid nodules with positron emission tomography," Surgery, vol. 114, no. 4, pp. 728-735, 1993.

[38] R. D. Utiger, "The multiplicity of thyroid nodules and carcinomas," The New England Journal of Medicine, vol. 352, no. 23, pp. 2376-2378, 2005.

[39] J. M. Kim, J. S. Ryu, T. Y. Kim et al., "18F-fluorodeoxyglucose positron emission tomography does not predict malignancy in thyroid nodules cytologically diagnosed as follicular neoplasm," Journal of Clinical Endocrinology and Metabolism, vol. 92, no. 5, pp. 1630-1634, 2007.

[40] R. V. Lloyd, L. A. Erickson, M. B. Casey et al., "Observer variation in the diagnosis of follicular variant of papillary thyroid carcinoma," American Journal of Surgical Pathology, vol. 28, no. 10, pp. 1336-1340, 2004.

[41] E. Gemsenjager, A. Perren, B. Seifert, G. Schüler, I. Schweizer, and P. U. Heitz, "Lymph node surgery in papillary thyroid carcinoma," Journal of the American College of Surgeons, vol. 197, no. 2, pp. 182-190, 2003.

[42] A. Frilling, K. Tecklenborg, R. Gorges, F. Weber, M. Clausen, and E. C. Broelsch, "Preoperative diagnostic value of $18 \mathrm{~F}$ fluorodeoxyglucose positron emission tomography in patients with radioiodine-negative recurrent well-differentiated thyroid carcinoma," Annals of Surgery, vol. 234, no. 6, pp. 804$811,2001$.
[43] W. Wang, H. Macapinlac, S. M. Larson et al., "[18F]-2-fluoro2-deoxy-D-glucose positron emission tomography localizes residual thyroid cancer in patients with negative diagnostic (131)I whole body scans and elevated serum thyroglobulin levels," Journal of Clinical Endocrinology and Metabolism, vol. 84, no. 7, pp. 2291-2302, 1999.

[44] G. Lal, T. Fairchild, J. R. Howe, R. J. Weigel, S. L. Sugg, and Y. Menda, "PET-CT scans in recurrent or persistent differentiated thyroid cancer-is there added utility beyond conventional imaging?" Surgery, vol. 148, no. 6, pp. 1082-1089, 2010.

[45] T. Petrich, A. R. Borner, D. Otto, M. Hofmann, and W. H. Knapp, "Influence of rhTSH on (18F) fluorodeoxyglucose uptake by differentiated thyroid carcinoma," European Journal of Nuclear Medicine, vol. 29, no. 5, pp. 641-647, 2002.

[46] F. Moog, R. Linke, N. Manthey et al., "Influence of thyroidstimulating hormone levels on uptake of FDG in recurrent and metastatic differentiated thyroid carcinoma," Journal of Nuclear Medicine, vol. 41, no. 12, pp. 1989-1995, 2000.

[47] B. B. Chin, P. Patel, C. Cohade, M. Ewertz, R. Wahl, and P. Ladenson, "Recombinant human thyrotrophin stimulation of fluoro-D-glucose positron emission tomography uptake in well-differentiated thyroid carcinoma," Journal of Clinical Endocrinology and Metabolism, vol. 89, no. 1, pp. 91-95, 2004.

[48] B. F. Branstetter IV, T. M. Blodgett, L. A. Zimmer et al., "Head and neck malignancy: is PET/CT more accurate than PET or CT alone?” Radiology, vol. 235, no. 2, pp. 580-586, 2005.

[49] A. Razfar, B. F. Branstetter IV, A. Christopoulos et al., "Clinical usefulness of positron emission tomography-computed tomography in recurrent thyroid carcinoma," Archives of Otolaryngology_Head and Neck Surgery, vol. 136, no. 2, pp. 120-125, 2010. 


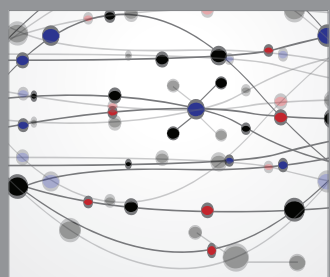

The Scientific World Journal
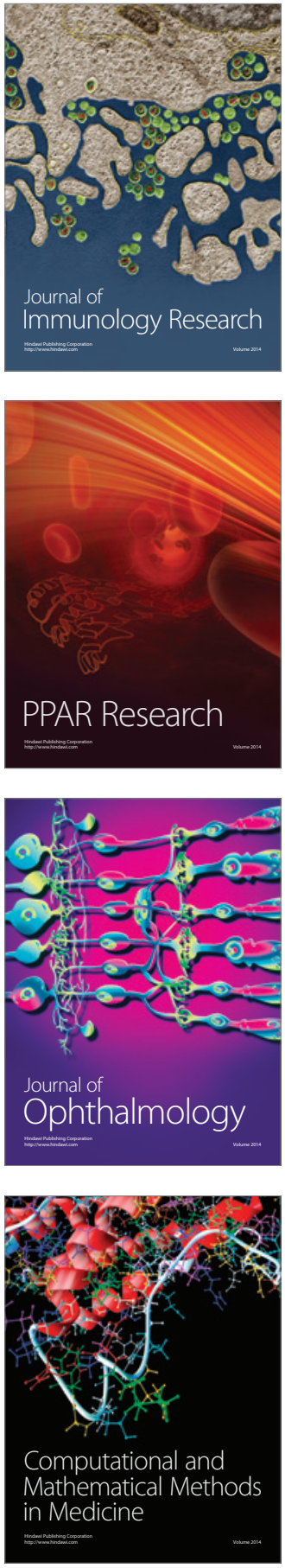

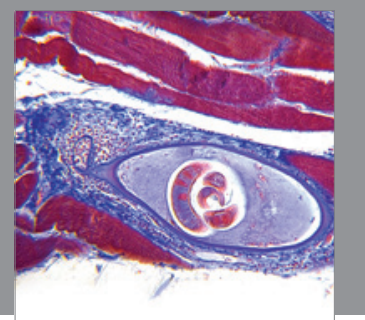

Gastroenterology

Research and Practice
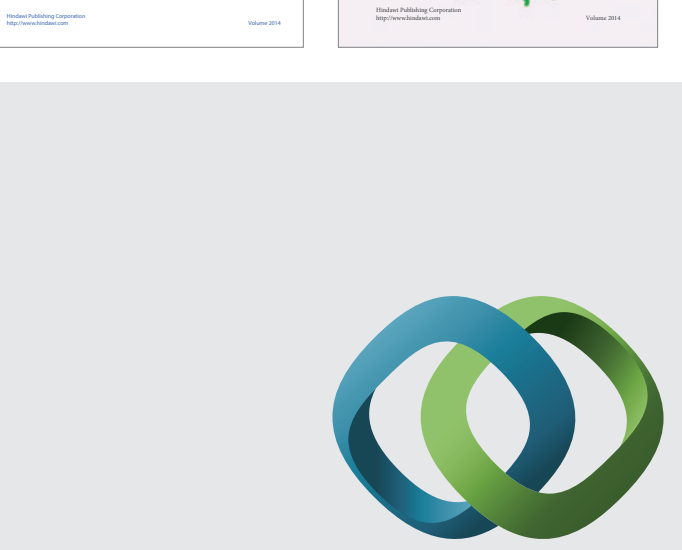

\section{Hindawi}

Submit your manuscripts at

http://www.hindawi.com
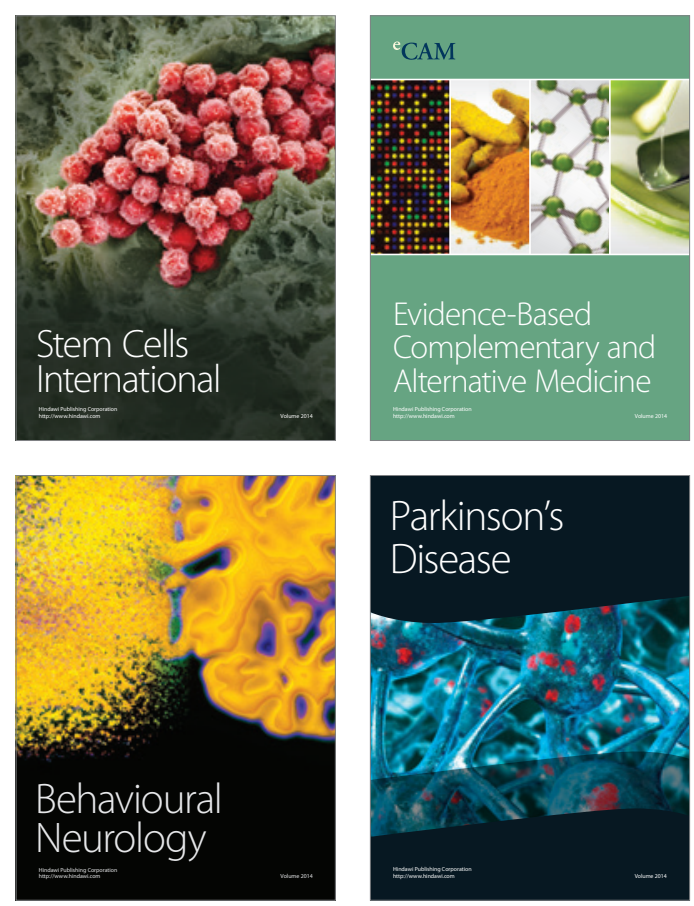

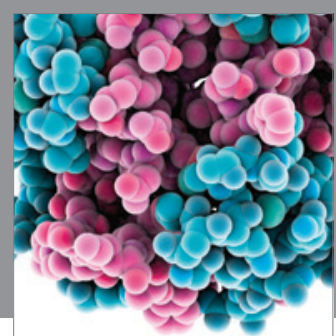

Journal of
Diabetes Research

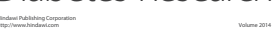

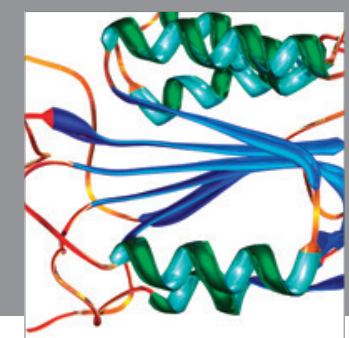

Disease Markers
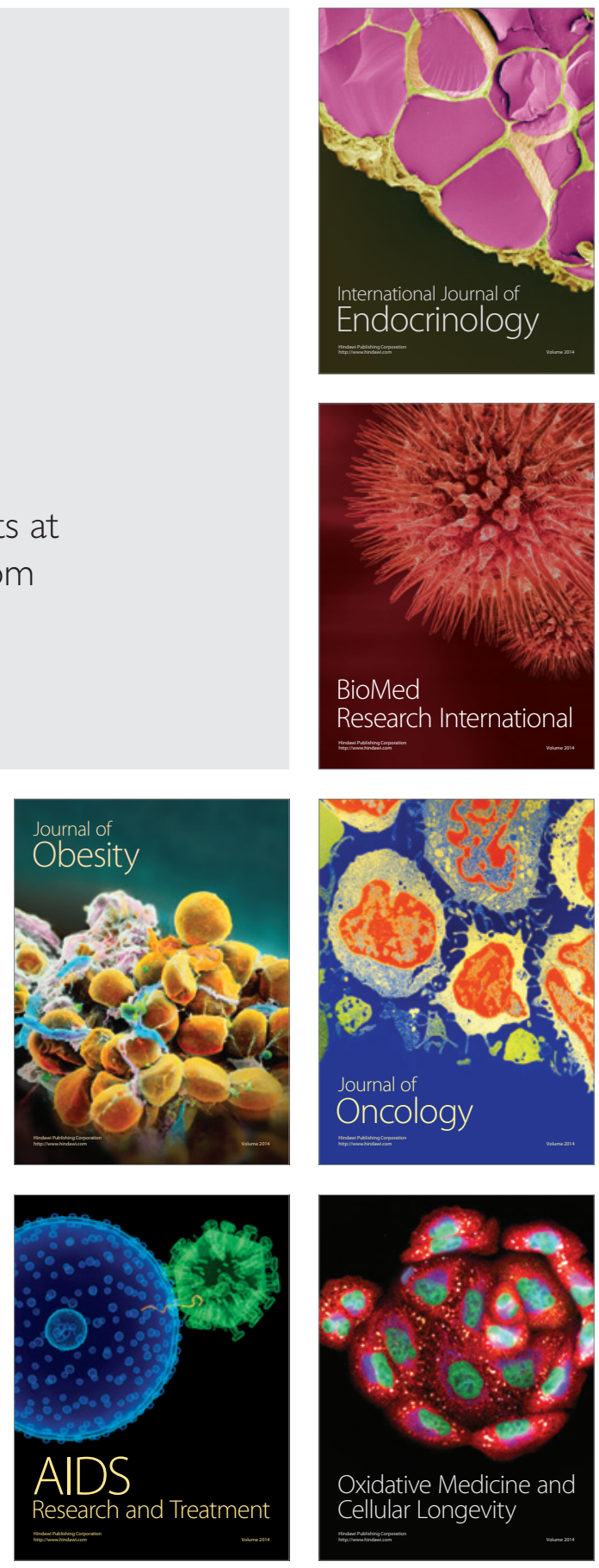